\title{
Simulation Investigation on Application of Powder Metallurgy Damping Effect in Water Hydraulic Pump
}

\author{
Weijie Shi, Xiaohui Luo, Zuti Zhang, Huawei Wang and Yuquan Zhu \\ School of Mechanical Science and Engineering, Huazhong University of Science and Technology, Wuhan, 430074, China
}

\begin{abstract}
Over the years, the hydrostatic balance slipper is often used to make the hydraulic lubrication film formed between the swash plate and the slipper. In this paper, a slipper containing the powder metallurgy (PM) composite material is proposed. The porous characteristics have an automatic damping effect, which can improve the tribological characteristics of the slipper. Based on the Reynolds equation, porous Darcy law and continuity equation, a lubrication model of PM damper is proposed to analyze the damping effect of PM in this paper. The proposed model is calculated using the finite volume method to capture the water film characteristics. Effects of length, diameter and porosity of the PM damper on the water film thickness are investigated. The results show that the water film can be formed by adopting the PM damper. In order to ensure the slipper with PM damper to form hydrostatic support, the length, diameter and porosity of PM damper is recommended to be $5 \mathrm{~mm}, 8 \mathrm{~mm}$ and 0.7 , respectively.
\end{abstract}

\section{Introduction}

Water hydraulics system has been of great interest to researchers because of its environmental friendliness, easy disposal and safety. As a crucial component in the hydraulic system, axial piston pumps are widely used for power density, high efficiency and structure compactness. Swash plate-slipper is one of the most important friction pairs in the hydraulic axial piston pumps and it plays a key role in the pump's performance. However, the low viscosity and poor lubrication of water make it difficult to form the water lubrication film, which can produce boundary friction or even unlubrication friction [1]. Therefore, it is essential to study the lubrication characteristics of the swash plate-slipper friction pair.

Hydrostatic slipper bearing with the damping hole is an effective way to maintain a fluid film between swash plate and slipper. Previously, many researches on the damping hole were conducted via simulation and experiment. E. Koç and C. J. Hooke [2] examined the design of hydrostatically balanced bearings as used in the slippers of high pressure axial piston pumps, and outlined a design procedure whereby the slipper behaviour. It is shown that for successful operation the slippers need to have small amounts of non-flatness on the running surfaces. Researchers at the Maha Fluid Power Research Center have developed a simulation software CASPAR to simulate the pump performances. In particular, CASPAR can predict the lubrication performance between the slipper and swash plate [3, 4]. S. Kumar et al. [5] have investigated the characteristics of a piston pump slipper with a groove. The orifice radius and groove width are all $1 \mathrm{~mm}$. A test rig was built to compare experimental and
CFD results, finding a good agreement for all of the cases studied. In summary, water film is thinner than oil film between the swash plate and the slipper in piston pump.

On the other hand, the powder metallurgy (PM) material has excellent tribological properties and the most special place of PM is that it contains pores. The porosity can be controlled by adjusting the sintering process. A.G. Kostornovin et al. [6] prepared a series of bronze PM at the pressing pressure of $100-700 \mathrm{MPa}$, sintering temperature of by $770-860{ }^{\circ} \mathrm{C}$ and powder diameter of 1 $200 \mu \mathrm{m}$. They found that the porosity decreases with the increase of pressure and sintering temperature, and the fluctuation range of porosity is $11 \%-50 \%$. D. Gu [7] used the direct metal laser sintering to consolidate $\mathrm{Cu}$-based alloy powder reinforced with $\mathrm{Ni}$ particles and found that a high densification level of $95.2 \%$ theoretical density was obtained after sintering. G Sethi et al. [8] studied the sintering behaviour of the $\mathrm{Cu}-12 \mathrm{Sn}$ bronze system in both, a microwave furnace as well as a conventional furnace. They found that the densification degree of a microwave furnace is greatly improved.

In view of the porous properties, the PM is installed into the slipper. The damping effect of PM damper on the lubrication performance is investigated.

\section{Simulation methods}

\subsection{Damping effect model}

Figure 1 illustrates the structure and working principle of the damping effect. The PM is installed in the slipper. Similar to the damping hole, the water can flow across 
the PM into the water chamber to form the water film reaction force. The reaction force lifts the slipper to avoid its contact with the swash plate. When the cylinder pressure is increased, the retain force is raised and the water film thickness is decreased accordingly. On the other hand, the flow rate through the PM damper decreases with decreasing the water film thickness. Further, with decreasing the flow rate through the PM damper, the pressure in water chamber increases, which can balance the retain force.
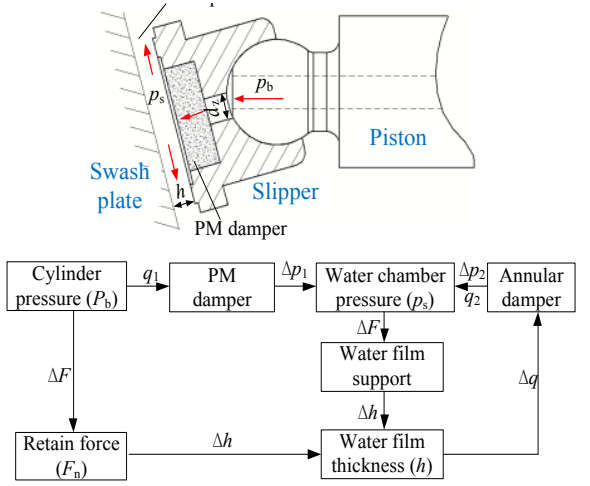

Figure 1. Structure and working principle of damping effect.

Assuming that the porosity of PM distributes uniformly, the flow velocity through the slipper can be calculated by the Darcy law

$$
U=\frac{K}{\eta} \nabla p
$$

where $K$ is the permeability of the $\mathrm{PM}$ and it can be calculated by

$$
K=\frac{\varphi^{3} d_{\mathrm{p}}^{2}}{150(1-\varphi)^{2}}
$$

The water in channels between tows is governed by the continuity equation:

$$
\operatorname{div}(U)=0
$$
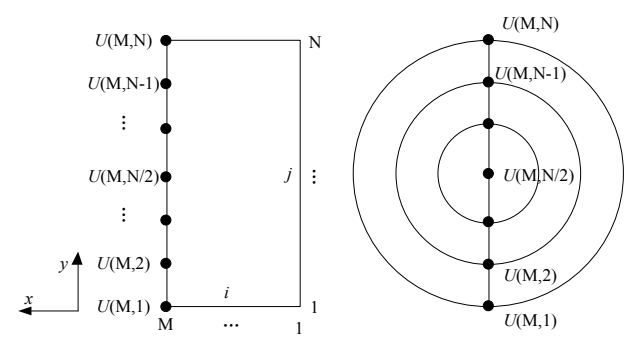

Figure 2. Calculation method of flow rate through the PM.

Figure 2 illustrates the calculation method of flow rate across the PM damper. The area of PM damper in the $x$ and $y$ direction is discretized using the finite difference mesh method with the $x$ step $\Delta x=l \mathrm{x} / M$ and $y$ step $\Delta y=l \mathrm{y} / N$. Assuming that the Darcy velocity at the outlet of PM damper is equal at the same radius, the flow rate through the slipper can be calculated by

$$
q_{1}=\sum_{j=1}^{\mathrm{N} / 2} U(M, j) \times 2 \pi j \Delta y^{2}
$$

The pressure of water chamber between the slipper and the swash plate is calculated by the characteristic equation of annular damper.

$$
q_{2}=\frac{\pi h^{3}\left(p_{\mathrm{s}}-p_{0}\right)}{6 \eta \ln \left(R_{0} / r_{0}\right)}
$$

The water through the slipper by the damper effect will flow through the annual damper into the chamber. According to the flow continuity equation, it can be concluded that the flow rate through the PM damper is equal to that through the annual damper.

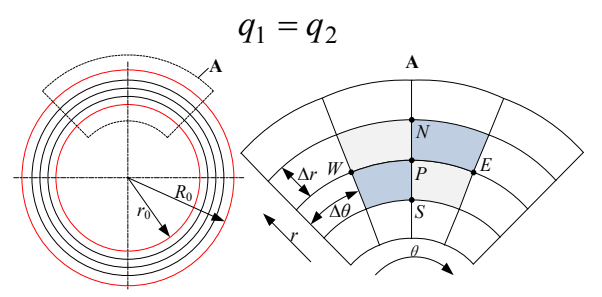

Figure 3. Mesh partition of finite volume method.

\subsection{Force characteristic model}

In the simulation, the piston force generated by the water pressure is only considered and it can be expressed as follows

$$
F_{\mathrm{b}}=\frac{\pi}{4} d^{2} P_{\mathrm{b}} / \cos \gamma
$$

Assuming that the pressure under the slipper at arbitrary point is $p(r, \theta)$, the water film support force generated by it can be described by

$$
F_{\mathrm{h}}=p_{\mathrm{s}} A_{\mathrm{s}}+\iint p r d r d \theta
$$

According to force equilibrium law, the force generated by water pressure is equal to the force generated by the water film:

$$
F_{\mathrm{b}}=F_{\mathrm{h}}
$$

\subsection{Water film pressure field}

To describe the water film pressure field of slipper friction pair, the Reynolds equation [9] is given as shown below

$$
\frac{1}{r} \frac{\partial}{\partial r}\left(r \frac{h^{3}}{\eta} \frac{\partial p}{\partial r}\right)+\frac{1}{r^{2}} \frac{\partial}{\partial \theta}\left(\frac{h^{3}}{\eta} \frac{\partial p}{\partial \theta}\right)=6 v_{\mathrm{sr}} \frac{\partial h}{\partial r}+6 v_{\mathrm{s} \theta} \frac{\partial h}{\partial \theta}+12 \frac{\partial h}{\partial t}
$$

The boundary conditions are the important prerequisite for solving Reynolds equation, which is illustrated in Eq. (11).

$$
\left\{\begin{array}{l}
p(r, 0)=p(r, 2 \pi),\left.\frac{\partial p}{\partial \theta}\right|_{(r, 0)}=\left.\frac{\partial p}{\partial \theta}\right|_{(r, 2 \pi)} \\
p\left(r_{0}, \theta\right)=p_{\mathrm{s}}, \quad p\left(R_{0}, \theta\right)=p_{0}
\end{array}\right.
$$

The water film thickness varying with time is ignored $(\partial h / \partial t=0)$. The Reynolds equation is an elliptic partial differential equation, which can be transformed into the linear equations by numerical discretization. According to the discrete theorem, the bottom sealing belt of slipper is conducted the mesh partition, as shown in Figure 3. Adopting the finite volume method with radius and angle steps respectively equal to $\Delta r=(R-r) / U$ and $\Delta \theta=2 \pi / V$, a linear equation is acquired 


$$
\begin{aligned}
& \left(r_{N} \frac{h_{N}^{3}}{\eta} \frac{p_{N}-p_{P}}{\Delta r}-r_{N} \frac{h_{N}^{3}}{\eta} \frac{p_{P}-p_{S}}{\Delta r}\right) \times 2 \Delta \theta \\
& +\left(\frac{1}{r_{E}} \frac{h_{E}^{3}}{\eta} \frac{p_{E}-p_{P}}{\Delta \theta}-\frac{1}{r_{W}} \frac{h_{W}^{3}}{\eta} \frac{p_{P}-p_{W}}{\Delta \theta}\right) \times 2 \Delta r \\
& =\left(6 v_{s r} r\right)_{P}\left(h_{N}-h_{S}\right) \times 2 \Delta \theta+\left(6 v_{s \theta}\right)_{P}\left(h_{E}-h_{W}\right) \times \frac{r_{N}^{2}-r_{S}^{2}}{2}
\end{aligned}
$$

Gauss-Seidel over-relaxation iteration method is adopted to solve the linear equation. In order to judge whether the iteration result meets the precision requirement, the relative error of pressure is defined

$$
\left|\frac{\sum p_{P}^{k+1}-\sum p_{P}^{k}}{\sum p_{P}^{k+1}}\right|<\delta
$$

where $\delta$ the relative error, $\delta=0.001$.

\subsection{Water film pressure field}

Figure 4 shows the coupling process of the simulation. Initially, the initial water film thickness $h_{0}$ and the slipper with the PM damper are given. Adopting the water film thickness as an input, the water film pressure field can be acquired by solving the Reynolds equation and the damping effect model. To avoid the case of negative pressure, a negative pressure is equal to zero. The water film support force is calculated and the force equilibrium equations are solved. When the force imbalance occurs, the water film thickness is adjusted. If the force balance occurs, the simulation terminates. Finally, the water chamber pressure, water film pressure field and water film thickness are acquired.

\begin{tabular}{|c|c|c|}
\hline Category & Parameter & Value \\
\hline \multirow{5}{*}{$\begin{array}{l}\text { Geometrical } \\
\text { parameters }\end{array}$} & Swash plate angle, $\gamma\left({ }^{\circ}\right)$ & 15 \\
\hline & $\begin{array}{l}\text { Inside radius of the slipper } \\
\text { sealing surface, } r_{0}(\mathrm{~m})\end{array}$ & 0.01 \\
\hline & $\begin{array}{l}\text { Outer radius of the slipper } \\
\text { sealing surface, } R_{0}(\mathrm{~m})\end{array}$ & 0.013 \\
\hline & Pitch radius of cylinder, $R(\mathrm{~m})$ & 0.043 \\
\hline & Diameter of the piston, $d(\mathrm{~m})$ & 0.023 \\
\hline \multirow{2}{*}{$\begin{array}{l}\text { Operating } \\
\text { parameters }\end{array}$} & Chamber pressure, $p_{\mathrm{b}}(\mathrm{MPa})$ & 14 \\
\hline & Rotation speed, $n(\mathrm{r} / \mathrm{min})$ & 750 \\
\hline PM parameters & Porosity of the PM, $\varphi(-)$ & 0.25 \\
\hline
\end{tabular}

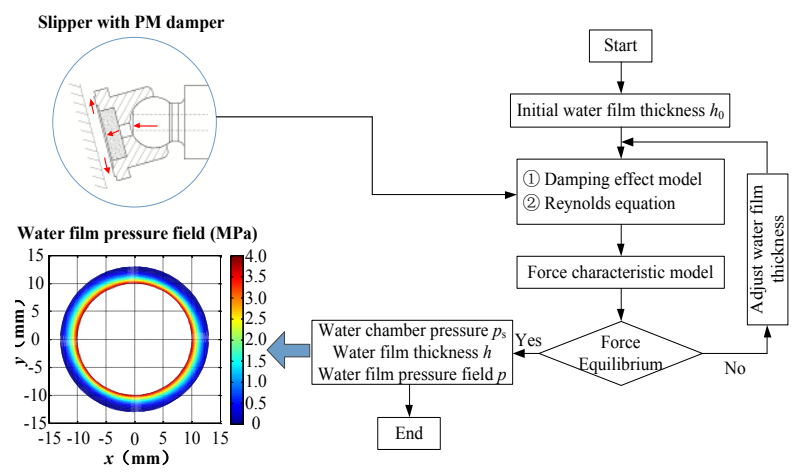

Figure 4. Coupling process of simulation.

Table 1. Parameters used in the simulation.

\section{Simulation results}

\subsection{Damping effect of PM}

The parameters used in the simulation are shown in Table 1, including geometrical parameters, operating parameters and PM parameters. Damping effect of PM is investigated at piston pressure of $14 \mathrm{MPa}$. When analyzing the pressure variation with porosity, diameter and length respectively equal to $0.7,8 \mathrm{~mm}$ and $10 \mathrm{~mm}$, the pressure is shown to be symmetric in angular direction due to the parallelism between swash plate and slipper. The results found by simulation are shown in Figure 5(a). The pressure decreases from inside out, because the angular force can produce movement of flow in angular direction. Figure 5(b) illustrates a twodimensional cross sectional cut through $y=0$ position of Figure 5(a). It can be seen that the value of the pressure along the water chamber is approximately constant $(13.69 \mathrm{MPa})$ and the pressure loss is $0.31 \mathrm{MPa}$. It indicates that the water can flow through the PM damper which generates the pressure loss. The pressure distributes uniformly along the sealing surface and the growth rate from external to internal is a constant $(4.64 \mathrm{MPa} / \mathrm{mm})$. In addition, the water film thickness is about $7.41 \mu \mathrm{m}$. It can be concluded that water film lubrication can be formed with adopting slipper installed the PM damper when the parameter is reasonably designed.
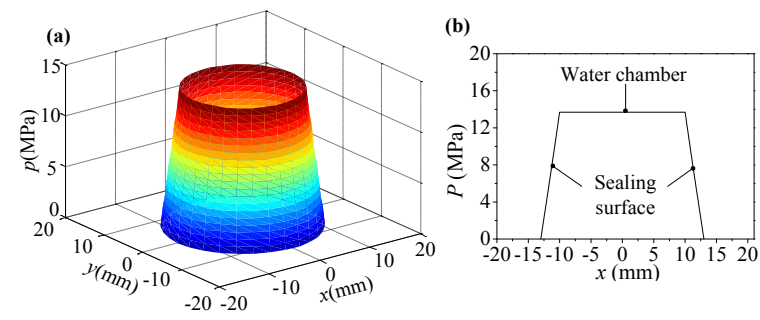

Figure 5. Pressure distribution of sealing surface under piston pressure of $14 \mathrm{MPa}$.

\subsection{Effect of length on the water film thickness}

According to the Darcy law, the length of PM damper has great influence on the water velocity into changing the leakage. Figure 6 illustrates the effect of water film thickness at different porosity. As shown in the figures, the water film thickness varies with the length of PM damper. When the length of PM damper increases, the water film thickness will decrease, which indicates that the lubrication becomes poor. In particular, the water film thickness is less than $2 \mu \mathrm{m}$ when the porosity is 0.1 . In addition, at different length of PM damper, the attenuation velocity of the water film thickness with increasing length of PM damper is different. Taking Figure 6(a) as an example, the water film thickness will decrease rapidly when the length of PM damper is less than $6 \mathrm{~mm}$. But when the length of PM damper is larger than $6 \mathrm{~mm}$, the water film thickness begins to decreases slowly. The results indicate that the water film is sensitive to the lower length of PM damper. However, the lower length will decrease its strength of PM damper. 

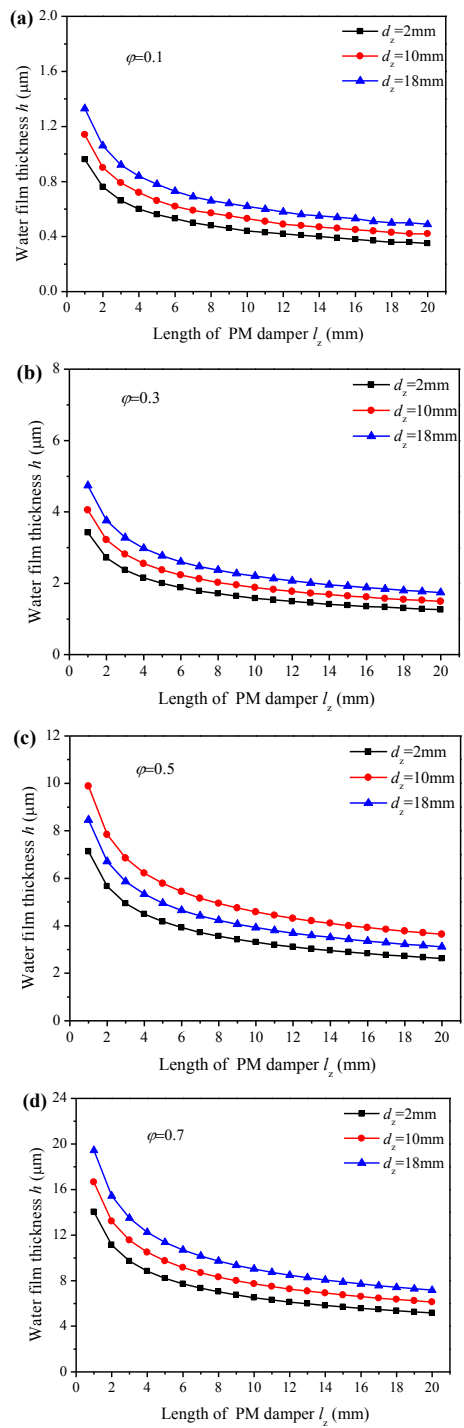

Figure 6. Effect of length on the water film thickness at different porosity: (a) $\varphi=0.1$, (b) $\varphi=0.3$, (c) $\varphi=0.5$, (d) $\varphi=0.7$.



Figure 7. Leakage varying with the length $(\varphi=0.5)$.

In addition, the leakage will be produced when the slipper with PM adopted. Effect of length of PM damper on the leakage at porosity of 0.5 is illustrated in Figure 6. The leakage is different at different length of PM damper. When the length of PM damper is raised, the leakage will reduce. The attenuation degree is divided into two parts: high attenuation and low attenuation. For example, at the diameter of PM damper of $18 \mathrm{~mm}$, the leakage decreases from $1.58 \mathrm{~L} / \mathrm{min}$ to $0.26 \mathrm{~L} / \mathrm{min}$ when the length of PM damper increases from $2 \mathrm{~mm}$ to $6 \mathrm{~mm}$. But when the length of PM damper is above $6 \mathrm{~mm}$, the leakage begins to decrease slowly and it maintains at $0.05 \mathrm{~L} / \mathrm{min}$.

\subsection{Effect of diameter on the water film thickness}

Figure 8 shows the relationship between the leakage and the diameter of PM damper. It can be seen that the water film thickness increases linearly with increasing diameter of PM damper, no matter what the porosity. As shown in the Figure 8(c), with the porosity and length of PM damper respectively equal to $0.5 \mathrm{~mm}$ and $2 \mathrm{~mm}$, the water film thickness will increase from $7.13 \mu \mathrm{m}$ to 9.88 $\mu \mathrm{m}$, when the length of PM damper increases from $1 \mathrm{~mm}$ to $18 \mathrm{~mm}$. The growth rate is $0.136 \mu \mathrm{m} / \mathrm{mm}$ and it is basically a constant. In order to ensure the contact area between the slipper and piston, the diameter of PM damper cannot be too large. For the slipper in this study, the diameter is considered to be smaller than $10 \mathrm{~mm}$.
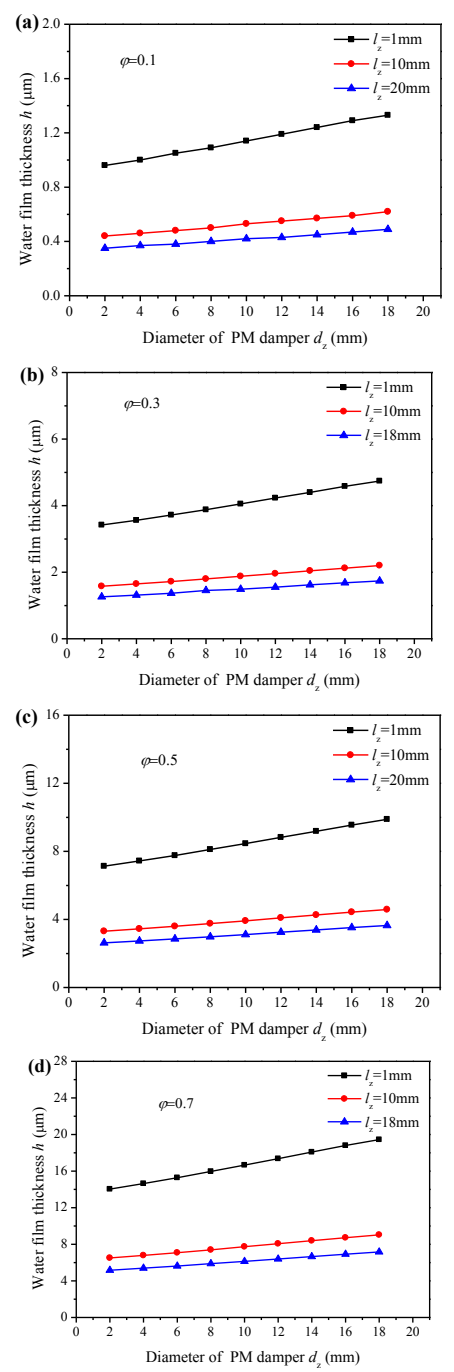

Figure 8. Effect of diameter on the water film thickness at different porosity: (a) $\varphi=0.1$, (b) $\varphi=0.3$, (c) $\varphi=0.5$, (d) $\varphi=0.7$.

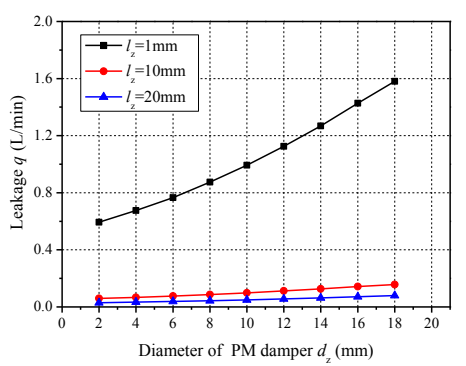

Figure 9. Leakage varying with the diameter $(\varphi=0.5)$. 
Additionally, the slipper leakage at porosity of 0.7 is simulated, which is shown in Figure 9. Different from the relationship between the water film thickness and the diameter of PM damper, the leakage does not increase linearly. The curve at the length of PM damper of $1 \mathrm{~mm}$ describes well this relationship in Figure 9.

\subsection{Effect of porosity on the water film thickness}

Porosity is an important parameter of PM damper. Considering the contact area and strength, the diameter of PM damper adopted in this study is $8 \mathrm{~mm}$. Effect of the porosity of PM damper on the water film thickness is described in Figure 10. It can be seen that the water film thickness increases nonlinearly with increasing porosity. At length of $2 \mathrm{~mm}$, the water film thickness increases from $0.87 \mu \mathrm{m}$ to $12.67 \mu \mathrm{m}$ when the porosity increases from 0.1 to 0.7 . Compared with the length and diameter of PM damper, the porosity has the highest effect on the water film thickness Also, the water film thickness will increase rapidly when the porosity is raised.

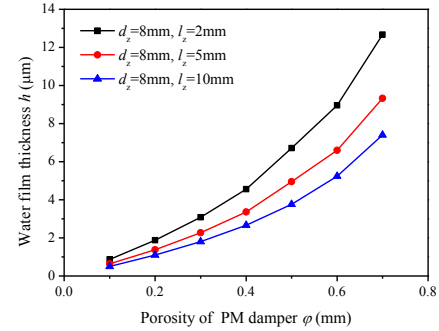

Figure 10. Effect of porosity on the water film thickness.

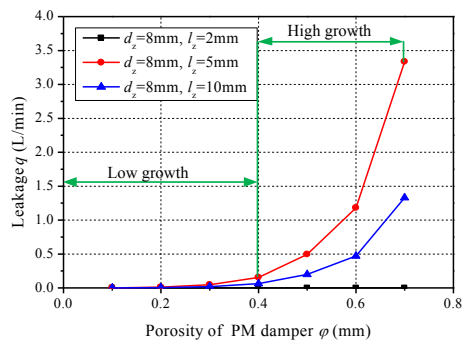

Figure 11. Leakage varying with the porosity $(\varphi=0.5)$.

In addition, the leakage varying with the porosity of PM damper is shown in Figure 11. As shown in Figure 11, the leakage increases with increasing porosity. But the growth rate is different at different porosity. When the porosity is less than 0.4 , the leakage is close to 0 . However, the leakage increases rapidly when the porosity is more than 0.4 . The growth rate is divided into high growth and low growth, which is illustrated in Figure 11. Considering the strength, lubrication and contact area, the length, diameter and porosity of PM damper is recommended to be $l_{\mathrm{z}}=5 \mathrm{~mm}, d_{\mathrm{z}}=8 \mathrm{~mm}$ and $\varphi=0.7$, respectively. The water film thickness is about $9.33 \mu \mathrm{m}$.

\section{Conclusion}

A slipper with PM damper is proposed in this paper. Based on the Reynolds equation, continuity equation and Darcy law, a lubrication model is built to analyze the damping effect of PM. The finite volume method is adopted to solve the equation. The results show that the water film can be produced with installing the PM damper. In addition, effects of length, diameter and porosity on the water film thickness and leakage are investigated. The water film and thickness decrease nonlinearly with increasing length of PM damper. On the other hand, the water film thickness increases linearly when the diameter of PM damper is raised. But the growth with increasing diameter is linear. Furthermore, the water film and thickness increase nonlinearly with increasing length of PM damper. As a result, in order to ensure the slipper with PM damper to form hydrostatic support, the parameters of PM damper is recommended to be $l_{\mathrm{z}}=5 \mathrm{~mm}, d_{\mathrm{z}}=8 \mathrm{~mm}$ and $\varphi=0.7$, respectively. Actually, the overturning phenomenon is occurred during the processing of slipper working. As a future work, the dynamic characteristics of slipper with PM damper can be investigated to analyze the damper effect of PM.

\section{Acknowledgment}

The authors acknowledge the Youth Science Foundation of National Natural Science Foundation of China (51305146).

\section{References}

1. B. Xu, Q. N. Wang, and J. H. Zhang, "Effect of case drain pressure on slipper/swashplate pair within axial piston pump," Journal of Zhejiang Universityence A, vol. 16, pp. 1001-1014, 2015.

2. E. Koç and C. J. Hooke, "Considerations in the design of partially hydrostatic slipper bearings," Tribology International, vol. 30, pp. 815-823, 1997.

3. A. Wondergem, "Piston / cylinder interface of axial piston machines - effect of piston micro-surface shaping," Dissertations \& Theses - Gradworks, 2014.

4. A. Schenk, "Predicting lubrication performance between the slipper and swashplate in axial piston hydraulic machines," Dissertations \& Theses Gradworks, 2014.

5. S. Kumar, J. M. Bergada, and J. Watton, "Axial piston pump grooved slipper analysis by CFD simulation of three-dimensional NVS equation in cylindrical coordinates," Computers \& Fluids, vol. 38, pp. 648-663, 2009.

6. A. G. Kostornov, "Tribological Characteristics of Materials Based on Bronze for Small Friction Assemblies," Powder Metallurgy and Metal Ceramics, vol. 42, pp. 379-382, 2003.

7. D. Gu, et. al, "Microstructural characteristics and formation mechanism of direct laser-sintered $\mathrm{Cu}$ based alloys reinforced with Ni particles," Materials \& Design, vol. 30, pp. 2099-2107, 2009.

8. G. Sethi, A. Upadhyaya, and D. Agrawal, "Microwave and conventional sintering of premixed and prealloyed $\mathrm{Cu}-12 \mathrm{Sn}$ bronze," vol. 35, pp. 264267, 2003.

9. T. Kazama, "Thermohydrodynamic Lubrication Model Applicable to a Slipper of Swashplate Type Axial Piston Pumps and Motors (Effects of Operating Conditions)," Tribology Online, vol. 5, pp. 250-254, 2010. 